\title{
Following Inhibition of BCL-2 by Antisense Oligonucleotides Compensatory Suppression of Apoptosis Involves the Direct Signal Transduction Pathway of LNCaP Cells
}

\author{
Marvin Rubenstein ${ }^{1,2,3,4^{*}}$, Courtney M. P. Hollowell4, Patrick Guinan ${ }^{1,3,4,5}$ \\ ${ }^{1}$ Division of Cellular Biology, Hektoen Institute for Medical Research, Chicago, IL, USA \\ ${ }^{2}$ Department of Biochemistry, Rush University Medical Center, Chicago, IL, USA \\ ${ }^{3}$ Department of Urology, Rush University Medical Center, Chicago, IL, USA \\ ${ }^{4}$ Division of Urology, Stroger Hospital of Cook County, Chicago, IL, USA \\ ${ }^{5}$ Department of Urology, University of Illinois at Chicago, Chicago, IL, USA \\ Email: ㅁMarv@Prodigy.net
}

Received 10 October 2014; revised 5 November 2014; accepted 20 November 2014

Copyright (C) 2015 by authors and Scientific Research Publishing Inc.

This work is licensed under the Creative Commons Attribution International License (CC BY). http://creativecommons.org/licenses/by/4.0/

(c) (i) Open Access

\begin{abstract}
Previously we have shown that when LNCaP cells are treated with antisense oligonucleotides (oligos) directed against BCL-2, compensatory changes in non-targeted genes take place in attempts to restore apoptosis and promote tumor aggressiveness. In addition to the inhibition of BCL-2, we find that the apoptosis promoter caspase-3 activity is suppressed, the transcription activity of STAT-3 is enhanced, while other regulators (bax, clusterin, AKT-1) associated with mitochondrial regulated apoptosis and caspase cascade are either unchanged or undetectable. We now evaluate proteins associated with the second pathway of apoptosis activation mediated by direct signal transduction involving fas, fas-ligand (a tumor necrosis factor-like cell surface receptor aka CD95), as well as the similar programmed death cell surface receptor (PD-1) and its respective ligand (PD-1L). This study evaluates the growth inhibition of in vitro propagating LNCaP cells employing mono- and bispecific oligos directed against BCL-2 [the second binding site was directed against the epidermal growth factor receptor (EGFR)]; and employing RT-PCR. The expression of these four proteins was evaluated. Expression of fas-ligand, PD-1 and PD-L1 were all significantly enhanced, whereas fas itself was undetectable. This suggests that in addition to pathways associated with the mitochondrial pathway of apoptosis, compensatory changes occur in the direct signal
\end{abstract}

*Corresponding author.

How to cite this paper: Rubenstein, M., Hollowell, C.M.P. and Guinan, P. (2015) Following Inhibition of BCL-2 by Antisense Oligonucleotides Compensatory Suppression of Apoptosis Involves the Direct Signal Transduction Pathway of LNCaP Cells. Open Journal of Apoptosis, 4, 1-10. http://dx.doi.org/10.4236/ojapo.2015.41001 
transduction pathway of this process. In addition to alterations in androgen sensitivity, growth factor expression and oncogene expression, these data suggest that suppressive BCL-2 therapy involves multiple pathways, including those involved with immune targeting and cytotoxicity and must be taken into account to make gene therapy more efficacious.

\section{Keywords}

Antisense Oligonucleotides, Prostate Cancer, Fas, Fas-Ligand, PD-1, PD-1 Ligand, Caspase-3, BCL-2, Bax, Therapy

\section{Introduction}

Antisense oligonucleotides (oligos) have been employed against both in vivo and in vitro prostate cancer model systems. For treatment, suppression has been directed towards protein growth factors, androgens (when oligos are directed against mRNA encoding 5-alpha reductase), receptors which bind either of these stimulating factors, inhibitors of apoptosis (BCL-2 [directly an inhibitor]; clusterin [an inhibitor of activated bax, which is a promoter]) and various oncogenes. Oligos provide a specific, relatively non-toxic method for translational arrest. While most assessments of activity quantitate the inhibition of in vitro growth, more specific methods, such as the polymerase chain reaction (PCR) are used to measure specific protein encoding.

Since most tumors are characterized by the enhanced expression of many proteins, growth is unlikely to be inhibited by knockdown of single gene products. Instead, multiple genes must be down regulated. Therefore, to increase oligo efficiency our lab evaluates bispecific derivatives [1]-[6] containing two binding sites on a single DNA oligo strand. We have shown the addition of a second binding site does not affect activity of the first and that dual binding sites can be directed against genes involved in either a single growth promoting autocrine loop [2] or towards those of different regulatory paths [3]-[6]. In these studies both mono- and bispecific oligos directed against BCL-2 were delivered by using a nanoparticle sized vehicle employing lipofectin.

Initial studies evaluated the effect of a single mono- and two bispecific oligos directed against BCL-2 by employing RT-PCR and measured the expression of both targeted BCL-2 and two untargeted apoptosis regulating proteins, bax and caspase-3. These proteins were chosen because they regulate apoptosis in opposing manners; BCL-2 is inhibitory, while activated bax and caspase-3 stimulate the process and each of these proteins regulates the mitochondrial mediated pathway. Results indicated that in addition to the expected specific suppression of BCL-2 the expression of caspase-3 was suppressed. Additional studies have shown that compensatory changes involve additional regulatory processes, including androgen sensitivity, growth factor and oncogene activity as well as transcriptional factors [7].

In this series of experiments we evaluated proteins associated with tumor cell destruction, via apoptosis, mediated via direct signal transduction. This is a process of initiating apoptosis through the binding of activating proteins (ligands) to cell surface receptors. When ligands bind to these receptors they activate a destructive cascade of protein interactions which lead to cell death. These receptors are structurally similar to the tumor necrosis factor receptor (CD95) and also regulate the immune system's cytotoxic T cell response. Therefore as mediators of the immune system their expression can have unanticipated effects on certain types of therapy since these proteins, particularly PD-1 and PD-L1, are now being targeted by monoclonal antibodies to treat cancer patients via immune checkpoint blockade.

If gene therapy is to be effective when directed against BCL-2, compensatory changes which compromise the effectiveness of the desired suppression must be identified and, if necessary for optimal anti-BCL-2 activity, additional proteins must be either suppressed or replaced.

\section{Methods}

\subsection{Oligonucleotides}

Oligos (mono- or bispecific) were purchased from Eurofins MWG Operon (Huntsville, AL, USA). Each was phosphorothioated on three terminal bases at 5' and 3' positions. Stock solutions were made to a final concentration of $625 \mu \mathrm{M}$ in sterile Dulbecco phosphate buffered saline (PBS). 
Base Sequences

Each oligo contained at least one CAT sequence and targeted the area adjacent to the AUG initiation codon for mRNA encoding the respective targeted protein (EGFR or BCL-2).

$\mathbf{M R}_{\mathbf{4}}$ (monospecific targeting BCL-2): T-C-T-C-C-C-A-G-C-G-T-G-C-G-C-C-A-T.

$\mathbf{M R}_{24}$ (bispecific targeting EGFR/BCL-2): G-A-G-G-G-T-C-G-C-A-T-C-G-C-T-G-C-T-C-T-C-T-C-C-C-AG-C-G-T-G-C-G-C-C-A-T.

$\mathbf{M R}_{42}$ (bispecific targeting BCL-2/EGFR): T-C-T-C-C-C-A-G-C-G-T-G-C-G-C-C-A-T-G-A-G-G-G-T-C-GC-A-T-C-G-C-T-G-C-T-C.

\subsection{Cell Culture}

LNCaP cells (American Type Culture Collection, Manassas, VA, USA) were grown in RPMI 1640 supplemented with $10 \%$ bovine serum, $1 \%$ L-glutamine and $1 \%$ penicillin/streptomycin in a $5 \% \mathrm{CO}_{2}$ incubator. Log phase cells were harvested by using EDTA/Trypsin and equally distributed into $75 \mathrm{~cm}^{2}$ flasks (Corning, NY, USA). At intervals media was either supplemented or replaced with fresh.

\subsection{Determination of Growth}

Four days prior to the addition of oligos $1 \times 10^{4} \mathrm{LNCaP}$ cells were added, in a total $200 \mu 1$ volume of media, to each depression of a 96-well plate and incubated at $37^{\circ} \mathrm{C}$ in a $5 \% \mathrm{CO}_{2}$ incubator. On the day of transfection, the following solutions were prepared:

1) $1 \mu \mathrm{l}$ of buffer containing either oligo or a diluent was added to $50 \mu \mathrm{l}$ of OPTI-MEM (Invitrogen Inc., Carlsbad, CA, USA) and gently mixed. One dilution was made for each well.

2) $1 \mu \mathrm{l}$ of lipofectin (Life Technologies Inc., Gaithersburg, MD, USA) was diluted in $50 \mu$ l of OPTI-MEM and mixed gently for 5 minutes at room temperature.

3) Oligo dilutions were mixed with $50 \mu \mathrm{l}$ of lipofectin and gently mixed for 20 minutes at room temperature.

4) $100 \mu \mathrm{l}$ of the lipofectin and oligo mixture was added to $100 \mu \mathrm{l}$ of RPMI medium and mixed.

Cells were incubated for 24 - 48 hours before solutions were aspirated and re-incubated for an additional 48 hrs in $200 \mu$ of media. Cell counts were determined following the addition of WST-1 reagent to each well, and after 2 hours the color intensity was measured by a microplate reader at a wavelength of $450 \mathrm{~nm}$, using a reference of $650 \mathrm{~nm}$. Values obtained were determined after the subtraction of paired blank samples from the experimental wells and were multiplied by a constant to give whole integers for analysis. Microsoft Excel software was utilized to calculate means and standard deviations, and Students t-tests were used to determine significance.

\subsection{Oligo Treatment Prior to PCR}

Four days prior to oligo addition, when cell density approached 75\% confluence, 10 ml of fresh media was added. Cells were incubated for an additional 3 days before $5 \mathrm{ml}$ of media was replaced with fresh the day before oligos were added. $100 \mu \mathrm{l}$ of stock oligos were added to bring the final concentration to $6.25 \mu \mathrm{M}$. Incubation proceeded for an additional 24 hours in the presence or absence of monospecific $\mathrm{MR}_{4}$, or the $\mathrm{MR}_{24} \mathrm{and}_{\mathrm{MR}}$ bispecific oligos.

\subsection{RNA Extraction}

Following treatment, media was removed, a single ml of cold $\left(4^{\circ} \mathrm{C}\right)$ RNAzol B was added to each $75 \mathrm{~cm}^{2}$ culture flask and the monolayer lysed by repeated passage through a pipette. All procedures were performed at $4^{\circ} \mathrm{C}$. The lysate was removed, placed in a centrifuge tube to which $0.2 \mathrm{ml}$ of chloroform was added, and shaken. The mixture stayed on ice for $5 \mathrm{~min}$, was spun at 12,000 $\times \mathrm{g}$ for $15 \mathrm{~min}$, and the upper aqueous volume removed and placed in a fresh tube. An equal volume of isopropanol was added, the tube shaken, and then allowed to stay at $4^{\circ} \mathrm{C}$ for 15 min before similar centrifugation to pellet the RNA. The supernatant was removed, the pellet washed in a single $\mathrm{ml}$ of $75 \%$ ethanol, then spun for $8 \mathrm{~min}$ at $7500 \times \mathrm{g}$. The ethanol was pipetted off and the formed pellet air dried at $-20^{\circ} \mathrm{C}$.

\subsection{RNA Quantitation}

RNA was resuspended in $250 \mu$ of diethylpyrocarbonate (DEPC) treated water (Invitrogen), and quantitated by 
using a Qubit Florometer and Quant-iT RNA Assay Kit (Invitrogen). DEPC is an inhibitor of RNase activity.

\subsection{RT-PCR}

Extracted RNA was diluted to $40 \mu \mathrm{g} / \mu \mathrm{l}$ in DEPC treated water. $1 \mu \mathrm{l}$ of this RNA was added to $1 \mu \mathrm{l}$ of both sense and antisense primers (forward and reverse sequences from Invitrogen) for human actin (used as a control) or 2 $\mu 1$ of combined primers for BCL-2, bax or caspase-3 (Real Time Primers, Elkins Park, PA). From a kit purchased from Invitrogen the following reactants were added for RT-PCR: $25 \mu \mathrm{l}$ of $2 \mathrm{x}$ reaction mixture, $2 \mu \mathrm{l}$ Super Script III RT/platinum Taq mix, tracking dye, and $\mathrm{MgSO}_{4}(3 \mu \mathrm{l}$ of a stock concentration of $5 \mathrm{mM}$, used for BCL-2, bax and caspase-3 vials only). DEPC treated water was added to yield a final volume of $50 \mu \mathrm{l}$. As a control for RT-PCR product production, human actin expression was tested in RNA extracted from HeLa cells which was provided in a kit purchased from Invitrogen. RT-PCR was performed for $2 \times 25$ cycles by using the F54 program in a Sprint PCR Thermocycler.

\section{Primers}

\section{1) Actin}

- Forward primer sequence: 5' CAA ACA TGA TCT GGG TCA TCT TCT C 3'.

- Reverse primer sequence: 5’ GCT CGT CGT CGA CAA CGG CTC.

- PCR product produced was 353 base pairs in length.

2) BCL-2

- Forward primer sequence: 5’ GAG ACA GCC AGG AGA AAT CA 3'.

- Reverse primer sequence: 5’ CCT GTG GAT GAC TGA GTA CC 3’.

- PCR product produced was 127 base pairs in length.

\section{3) Bax}

- Forward primer sequence: 5' GCT GGA CAT TGG ACT TCC TC 3’.

- Reverse primer sequence: 5' CTC AGC CCA TCT TCT TCC AG 3'.

- PCR product produced was 168 base pairs in length. 4) Caspase-3

- Forward primer sequence: 5' CCC CTG GAT CTA CCA GCA TA 3'.

- Reverse primer sequence: 5' TGT CTC TGC TCA GGC TCA AA 3'.

- PCR product produced was 262 base pairs in length. 5) Fas

- Forward primer sequence: 5’ TCA GTA CGG AGT TGG GGA AG 3'.

- Reverse primer sequence: 5' CAG GCC TTC CAA GTT CTG AG 3'.

- PCR product produced was 207 base pairs in length.

\section{6) Fas-ligand}

- Forward primer sequence: 5' GCA CTT TGG GAT TCT TTC CA 3'.

- Reverse primer sequence: 5' CCT CCA TTT GTC TGG CTC AT 3'.

- PCR product produced was 190 base pairs in length.

7) PD-1

- Forward primer sequence: 5' GAC TAT GGG GAG CTG GAT TT 3'.

- Reverse primer sequence: 5’ AGA GCA GTG TCC ATC CTC AG 3'.

- PCR product produced was 192 base pairs in length.

8) PD-L1

- Forward primer sequence: 5’ TGA TAC ACA TTT GGA GGA GAC G 3’.

- Reverse primer sequence: 5' CCC TCA GGC ATT TGA AAG TAT C 3'.

- PCR product produced was 261 base pairs in length.

\subsection{Detection and Quantitation of Product}

\subsubsection{Agarose Gel Electrophoresis}

$1.5 \%$ agarose gels were prepared in a $50 \mathrm{ml}$ volume of TBE buffer (1x solution: $0.089 \mathrm{M}$ Tris borate and 0.002 M EDTA, pH 8.3), containing $3 \mu \mathrm{l}$ of ethidium bromide $(10 \mathrm{mg} / \mathrm{ml}$ in 1x Tris borate buffer) in a Fisher Biotest electrophoresis system. Samples were run for 2 hours at a constant voltage of 70 by using a BioRad 1000/500 
power supply source. To locate the amplified PCR product, $3 \mu$ of a molecular marker which contained a sequence of bases in 100 base pair increments (Invitrogen) as well as $2 \mu \mathrm{l}$ of a sucrose based bromphenol blue tracking dye were run in each gel. For actin product localization, the tracking dye was included in each sample run; for all other products the tracking dye was run separately.

\subsubsection{Quantitation}

Gels were visualized under UV light and photographed by using a Canon 800 digital camera. Photographs were converted to black and white format and bands quantitated by using Mipav software provided by the National Institute of Health (NIH).

\section{Results}

\subsection{Cell Culture Experiments}

LNCaP cells were incubated with $\mathrm{MR}_{4}, \mathrm{MR}_{24}$ and $\mathrm{MR}_{42}$ and compared to lipofectin containing controls (Figure 1). In an initial experiment, each oligo significantly inhibited the growth of LNCaP cells: $\mathrm{MR}_{4}$ by $23.8 \%(\mathrm{p}=$ $0.0004) ; \mathrm{MR}_{24}$ by $31.2 \%$ ( $\left.<0.001\right)$; and $\mathrm{MR}_{42}$ by $31.7 \%$ ( $\left.<<0.001\right)$.

In a repeat experiment LNCaP cells were similarly incubated and compared to lipofectin containing controls. Bispecific oligos $\mathrm{MR}_{24}$, and $\mathrm{MR}_{42}$ produced significant respective inhibitions of $49.5 \%(\mathrm{p}<0.001)$ and $56.8 \%$ ( $\mathrm{p}$ $<0.001$ ), and were at least as effective as the mono-specific $\mathrm{MR}_{4}$ directed only towards BCL-2 in the inhibition of in vitro cell growth.

\subsection{RT-PCR Experiments}

When photographs of the identified product bands were scanned on agarose gels and quantitated using Mipav software, in a series of runs, the greatest expression of BCL-2 was always found in untreated LNCaP cells. Those treated with oligos, whether mono- or bispecific, produced bands which indicated obvious (to the naked eye) suppression. Figure 2 provides an example of one such band (actin) suitable for scanning and quantitation. The molecular weight markers shown in the left column are (in 100 base pair increments) 600 and 100 base pairs (from top to bottom). The band visualized between molecular markers of 300 and 400 base pairs is the expected 353 base pair human actin PCR product.

\subsubsection{BCL-2 Expression}

LNCaP cells incubated for 24 hours in the presence of $6.25 \mu \mathrm{M}$ of oligos demonstrated a suppression of BCL-2 expression, and support the finding of comparable biologic activity of both mono- and bispecific oligos seen in in vitro cell inhibition experiments. For each oligo evaluated, the greatest amount of suppression measured approached $100 \%$, for the mono-specific $\mathrm{MR}_{4}$; and for the bispecifics $\mathrm{MR}_{24}$ and $\mathrm{MR}_{42}, 86 \%$ and $100 \%$, respectively. Suppression was found in both repeat PCR runs with BCL-2 primers, as well as in repetitive agarose gel

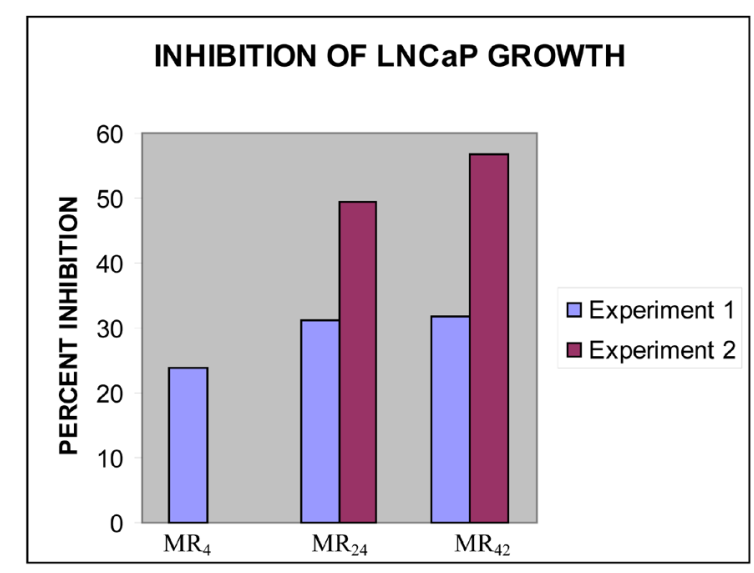

Figure 1. Inhibition of in vitro growth of LNCaP cells by mono- and bispecific oligos. 


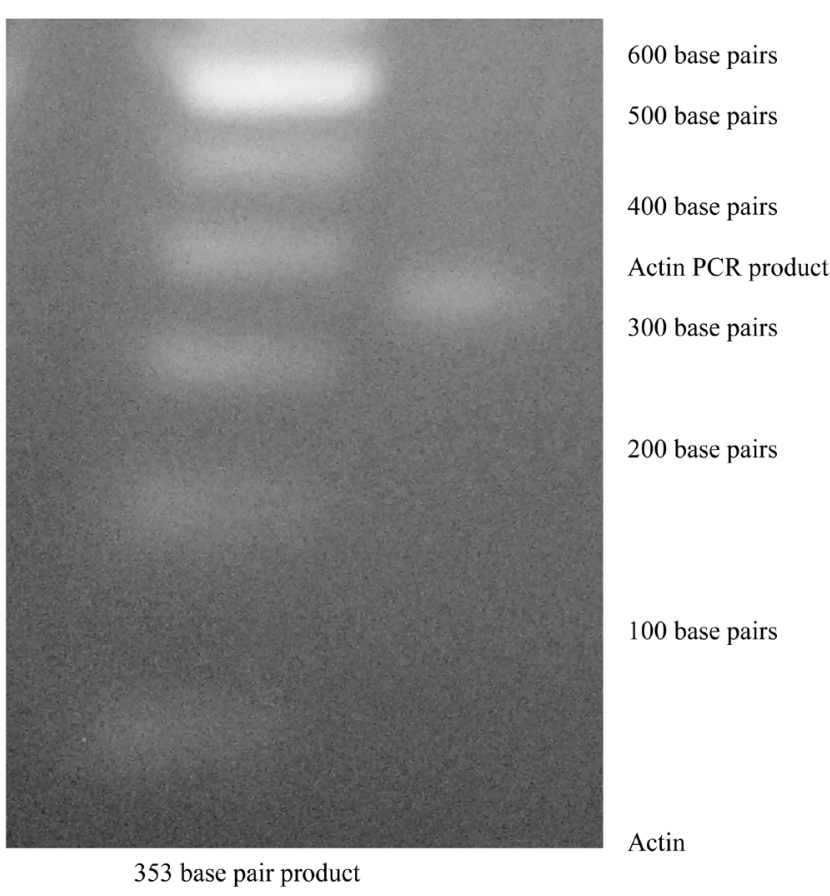

Figure 2. Expression of actin on agarose gel.

quantifications. Figure 3 presents a BCL-2 product band in the expected 127 base pair region which in this run was inhibited $23 \%$ by treatment with the mono-specific $\mathrm{MR}_{4}$, and $86 \%$ and $74 \%$, respectively by bispecifics $\mathrm{MR}_{24}$ and $\mathrm{MR}_{42}$, as measured by Mipav software.

Based upon both inhibition of cell growth and BCL-2 expression, we conclude that bispecific antisense oligos directed against EGFR and BCL-2, regardless of their tandem orientation, are at least as effective as the monospecific type directed solely towards BCL-2. The addition of a second mRNA binding site (directed towards EGFR) to these oligos does not prevent activity at the site specific for BCL-2.

\subsubsection{Bax Expression}

Identical amounts of extracted RNA from LNCaP cells treated with either mono- or bispecific oligos directed against BCL-2 (and EGFR in the bispecifics). This RNA was evaluated by RT-PCR using primers directed against bax. A representative band for bax is presented in Figure 4.

When background intensity was subtracted, the relative intensity of the bands corresponding to bax representing cells treated with $\mathrm{MR}_{4}, \mathrm{MR}_{24}$ and $\mathrm{MR}_{42}$ compared to controls were $-5.74 \% \pm 16.9 \%, 5.54 \% \pm$ $19.2 \%$, and $-15.34 \% \pm 32.9 \%$. These results were pooled from both duplicate PCR runs and gels and indicated that no significant differences in bax expression were found, compared to that seen with BCL-2.

\subsubsection{Caspase-3 Expression}

Identical amounts of extracted RNA from LNCaP cells treated with either mono- or bispecific oligos directed against BCL-2 (and EGFR in the bispecifics). This RNA was evaluated by RT-PCR using primers directed against caspase-3. A representative band for caspase-3 is presented in Figure 5 and appears immediately below the marker representing 300 base pairs.

When background intensity was subtracted, the relative intensity of the bands corresponding to caspase- 3 representing cells treated with $\mathrm{MR}_{4}, \mathrm{MR}_{24}$ and $\mathrm{MR}_{42}$ compared to controls were $-35.8 \% \pm 12.5 \%(\mathrm{p}=0.0002)$, $-40.3 \% \pm 16.6 \%(\mathrm{p}=0.0006)$ and $-43.5 \% \pm 26.3 \%(\mathrm{p}=0.006)$. These results were pooled from both duplicate PCR runs and gels and indicated that similar significant suppression of caspase-3 activity was demonstrated with each oligo (and type) evaluated.

\subsubsection{Fas Expression}

Identical amounts of extracted RNA from LNCaP cells treated with either mono- or bispecific oligos directed 


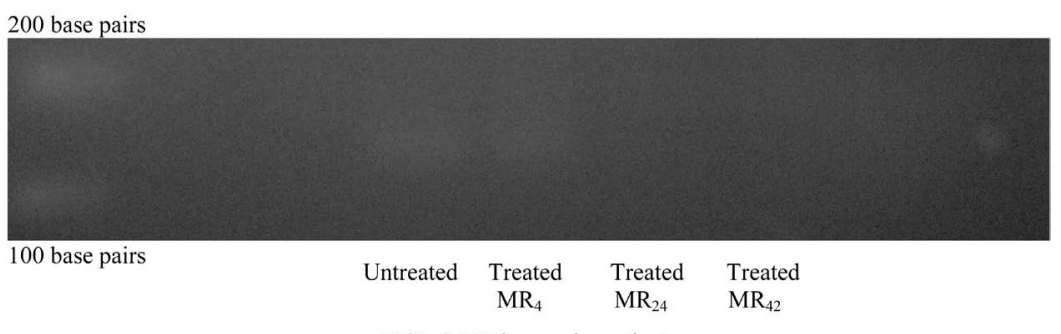

BCL-2 127 base pair product

Figure 3. Expression of BCL-2 on agarose gel.

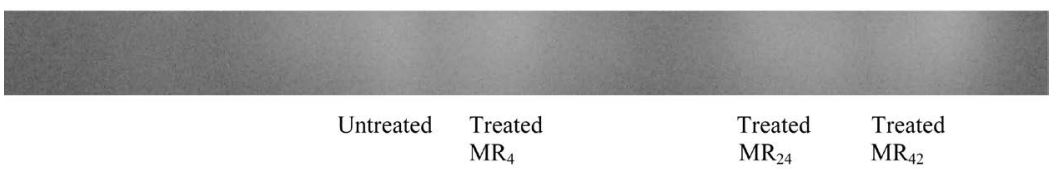

Bax is a 168 base pair product

Figure 4. Expression of Bax on agarose gel.

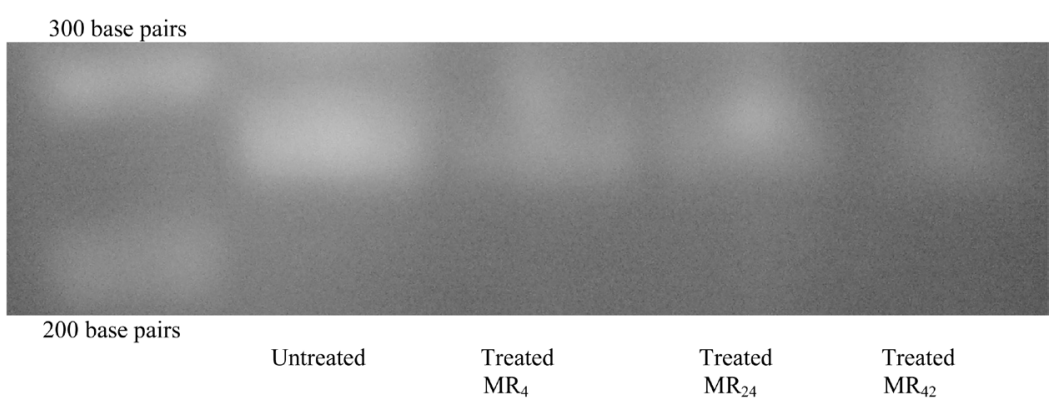

Caspase- 3 is a 262 base pair product

Figure 5. Expression of caspase-3 on agarose gel.

against BCL-2 (and EGFR in the bispecifics). This RNA was evaluated by RT-PCR using primers directed against fas. Expression of fas was undetectable in any of the treated or untreated LNCaP cells.

\subsubsection{Fas Ligand Expression}

Identical amounts of extracted RNA from LNCaP cells treated with either mono- or bispecific oligos directed against BCL-2 (and EGFR in the bispecifics). This RNA was evaluated by RT-PCR using primers directed against fas ligand. A representative band for fas ligand is presented in Figure 6. When background intensity was subtracted, the relative intensity of the bands corresponding to fas ligand representing cells treated with $\mathrm{MR}_{4}$, $\mathrm{MR}_{24}$ and $\mathrm{MR}_{42}$ compared to controls were increased $88.6 \% \pm 24.2 \%(\mathrm{p}=0.00033), 66.7 \% \pm 39.6 \%(\mathrm{p}=$ $0.015039)$ and $75.6 \% \pm 28.4 \%(\mathrm{p}=0.001784)$. These indicated that similar significant enhancement of fas ligand activity was demonstrated with each oligo (and type) evaluated.

\subsubsection{PD-1 Expression}

Identical amounts of extracted RNA from LNCaP cells treated with either mono- or bispecific oligos directed against BCL-2 (and EGFR in the bispecifics). This RNA was evaluated by RT-PCR using primers directed against PD-1. A representative band for PD-1 is presented in Figure 7. When background intensity was subtracted, the relative intensity of the bands corresponding to PD-1 representing cells treated with $\mathrm{MR}_{4}, \mathrm{MR}_{24}$ and $\mathrm{MR}_{42}$ compared to controls were increased $149.3 \% \pm 113.3 \%(\mathrm{p}=0.018476), 320.7 \% \pm 281.0 \%(\mathrm{p}=0.034078)$ and 193.9\% $\pm 117.0 \%(\mathrm{p}=0.005988)$. These indicated that similar significant enhancement of PD-1 activity was demonstrated with each oligo (and type) evaluated.

\subsubsection{PD-1 Ligand (PD-L1) Expression}

Identical amounts of extracted RNA from LNCaP cells treated with either mono- or bispecific oligos directed 


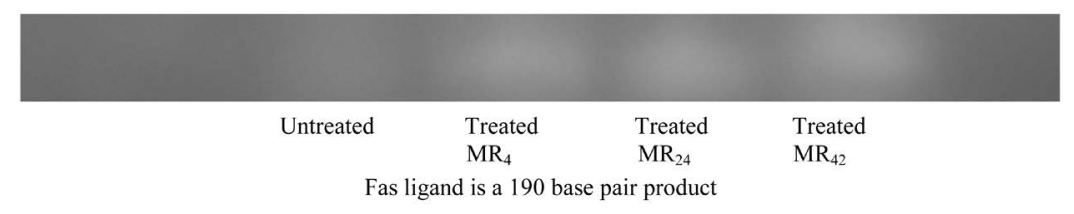

Figure 6. Expression of Fas ligand on agarose gel.

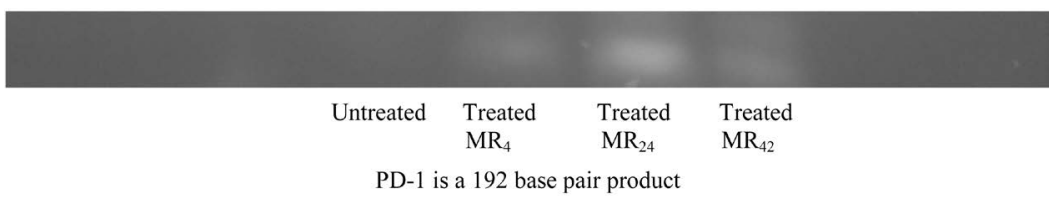

Figure 7. Expression of PD-1 on agarose gel.

against BCL-2 (and EGFR in the bispecifics). This RNA was evaluated by RT-PCR using primers directed against PD-L1. A representative band for PD-1L is presented in Figure 8. When background intensity was subtracted, the relative intensity of the bands corresponding to PD- $1 \mathrm{~L}$ representing cells treated with $\mathrm{MR}_{4}, \mathrm{MR}_{24}$ and $\mathrm{MR}_{42}$ compared to controls were increased 33.3\% $\pm 20.1 \%(\mathrm{p}=0.006079), 51.0 \% \pm 36.7 \%(\mathrm{p}=0.014394)$ and $28.5 \% \pm 19.3 \%(\mathrm{p}=0.010795)$. These indicated a small but significant enhancement of PD-L1 activity was demonstrated with each oligo (and type) evaluated.

\section{Discussion}

Gene therapy for the treatment of cancer is a complex process, because for it to work several pathways (and their regulatory proteins) must be simultaneously regulated (or activities replaced). As such therapy becomes increasingly available and administered we find that it is not as protein specific as initially promoted. Due to sequence homology additional proteins can be affected, and as we continue to identify alternative splicing patterns for mRNA to yield different proteins, it becomes increasingly less specific as these new proteins, with their homologous sequences, are identified.

Clinically, gene therapy is now being administered which targets and suppresses some of the apoptotic inhibitory proteins, particularly BCL-2. These antisense oligos attempt to restore apoptosis associated with chemotherapy [8] and radiation treatment [9] and at least one is being evaluated against prostate cancer [10].

Previous reports from our group described how non-sequence related and non-targeted genes can alter their patterns of gene expression in attempts to become resistant to this treatment and re-establish apoptosis. In addition, we reported that tumors are able to further adapt through increased androgen sensitivity, growth factor production, and increased oncogene and transcription factor activity [7]. We now report an additional process by which the original targeted process of apoptosis can be affected, by the direct signal transduction pathway. This pathway is now being evaluated due to its role in immune regulation and cytotoxic T cell targeting. Not part of the mitochondrial pathway of apoptosis, the fas receptor is sometimes referred to as the death receptor, and regulates a second pathway for apoptosis mediated by direct signal transduction. When fas binds its ligand, it initiates a cascade of events culminating in cell death and removal of the debris. PD-1 (CD279) and its ligand are thought to negatively regulate an immune response through a process referred to as immune checkpoint blockade. Antibodies directed against them are now being administered to treat melanoma and other solid tumors. Although the degree to which several of these markers are altered may appear minimal, the differences are statistically significant. Those genes whose activity are adversely reduced (like caspase-3), may have to be either restored or their genes replaced for anti-BCL-2 treatment to be effective. In addition, we have suggested that antiBCL-2 therapy could make prostate tumors more androgen sensitive and inadvertently select a more aggressive phenotype [11]. Therefore these types of experiments provide important insight to tumor adaptability and call attention to factors which must be taken into consideration when gene therapy is contemplated, in order for it to be made more effective.

Tumor cells are individually heterogenous, and those which evade growth regulation are selected. In addition, variants, as their DNA becomes increasingly unstable, tend to accumulate additional adaptations (mutations) which further contribute to aggressiveness, resistance and dissemination. Treatment protocols administered to correct one genetic alteration can, through selective pressure, initiate compensatory changes which diminish the 


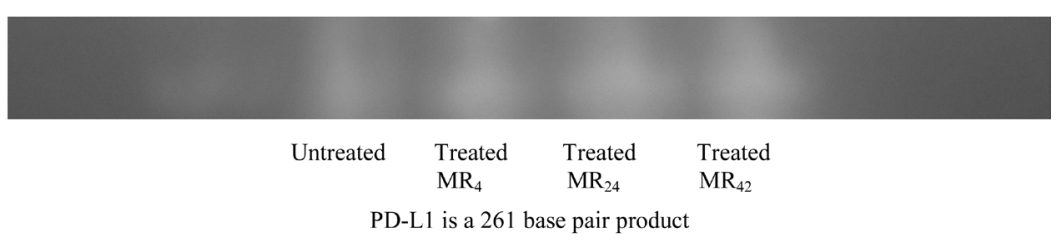

Figure 8. Expression of PD-L1 on agarose gel.

effectiveness of the original, and in many tumors, some of the early mutational events lead to evasion of apoptosis. This "programmed" process clears the body of altered (transformed) or damaged cells. It is highly regulated and involved many proteins being synthesized, recognized (via receptors) or otherwise interacting or activating each other. The overall efficiency of this process is regulated by many proteins, acting in multiple pathways; with individual pathways often regulated by the overall contribution by both stimulatory and inhibitory influences. Selection of cells which resist apoptosis is no different than the process by which hormone sensitive prostate tumor cells, in the absence of androgen, are selected (and establish themselves) as insensitive variants. Therefore, for suppressive gene therapy to work, it's important to identify compensatory effects and those pathways which are particularly involved.

In this study we summarized the effect of oligo mediated growth suppression on three regulators of mitochondrial mediated apoptosis: BCL-2 (an inhibitor), bax and caspase-3 (both promoters) and two cell surface, tumor necrosis factor like, receptors which regulate the direct signal transduction pathway through interaction with their respective ligands.

For gene therapy to be successful, effects on untargeted genes must be identified. This is particularly important when translational inhibitors are administered and directed against BCL-2. Should additional proteins (inhibitors of apoptosis) be indicated for suppression, bispecific oligos or even proposed multifunctional and branched derivatives could then be employed [12]. Employing oligos to treat prostate cancer was first suggested 20 years ago by our laboratory [13] and we were the first to provide evidence that EGFR would provide a suitable target for treating prostate cancer [14]. This is why the bispecific oligos employed in these studies are simultaneously directed against EGFR. However, much work remains to be done, including that in primary prostate cell lines. Although LNCaP is the most frequently utilized in vitro model for prostate cancer, primary cell lines could give a more representative perspective of how prevalent and reproducible these compensations are. Therefore additional studies are underway to identify altered expressions in other proteins associated with apoptosis.

\section{Acknowledgements}

The Cellular Biology Laboratory at the Hektoen Institute is supported, in part, by the Blum Kovler Foundation, the Cancer Federation, Safeway/Dominicks Campaign for Breast Cancer Awareness, Lawn Manor Beth Jacob Hebrew Congregation, the Max Goldenberg Foundation, the Sternfeld Family Foundation, and the Herbert C. Wenske Foundation.

\section{References}

[1] Rubenstein, M., Tsui, P. and Guinan, P. (2005) Construction of a Bispecific Antisense Oligonucleotide Containing Multiple Binding Sites for the Treatment of Hormone Insensitive Prostate Tumors. Medical Hypotheses, 65, 905-907. http://dx.doi.org/10.1016/j.mehy.2004.12.032

[2] Rubenstein, M., Tsui, P. and Guinan, P. (2006) Bispecific Antisense Oligonucleotides with Multiple Binding Sites for the Treatment of Prostate Tumors and Their Applicability to Combination Therapy. Methods and Findings in Experimental and Clinical Pharmacology, 28, 515-518. http://dx.doi.org/10.1358/mf.2006.28.8.1003571

[3] Rubenstein, M., Tsui, P. and Guinan, P. (2007) Combination Chemotherapy Employing Bispecific Antisense Oligonucleotides Having Binding Sites Directed against an Autocrine Regulated Growth Pathway and BCL-2 for the Treatment of Prostate Tumors. Medical Oncology, 24, 372-378. http://dx.doi.org/10.1007/s12032-007-0023-y

[4] Rubenstein, M., Tsui, P. and Guinan, P. (2009) Multigene Targeting of Signal Transduction Pathways for the Treatment of Breast and Prostate Tumors: Comparison between Combination Therapies Employing Bispecific Oligonucleotides with either Rapamycin or Paclitaxel. Medical Oncology, 26, 124-130.

http://dx.doi.org/10.1007/s12032-008-9088-5 
[5] Rubenstein, M., Tsui, P. and Guinan, P. (2007) Bispecific Antisense Oligonucleotides Having Binding Sites Directed against an Autocrine Regulated Growth Pathway and BCL-2 for the Treatment of Prostate Tumors. Medical Oncology, 24, 189-196. http://dx.doi.org/10.1007/BF02698039

[6] Rubenstein, M., Tsui, P. and Guinan, P. (2008) Treatment of MCF-7 Breast Cancer Cells Employing Mono- and Bispecific Antisense Oligonucleotides Having Binding Specificity toward Proteins Associated with Autocrine Regulated Growth and BCL-2. Medical Oncology, 25, 182-186. http://dx.doi.org/10.1007/s12032-007-9018-y

[7] Rubenstein, M., Hollowell, C.M.P. and Guinan, P. (2012) No Compensation in VEGF Expression Follows Antisense Suppression of BCL-2 Activity. In Vivo, 26, 937-940.

[8] Yamanaka, K., Miyake, H., Zangemeister-Wittke, U., Jansen, B. and Gleave, M. (2004) Novel Bispecific Antisense Oligonucleotides Inhibiting Both BCL-2 and BCL-xL Expression Induce Apoptosis and Enhance Chemosensitivity in Human Androgen-Independent Prostate Cancer Cells. Proceedings of the American Association for Cancer Research, 45 (online), Abstract \#2930.

[9] Yip, K.W., Mocanu, J.D., Billie Au, P.Y., Sleep, G.T., Huang, D., Busson, P., Yeh, W.-C., Gilbert, R., O’Sullivan, B., Gullane, P., Bastianutto, C. and Liu, F.F. (2005) Combination BCL-2 Antisense and Radiation Therapy for Nasopharyngeal Cancer. Clinical Cancer Research, 11, 8131-8144. http://dx.doi.org/10.1158/1078-0432.CCR-05-1266

[10] Mu, Z.M., Hachem, P. and Pollack, A. (2005) Antisense BCL-2 Sensitizes Prostate Cancer Cells to Radiation. The Prostate, 65, 331-340. http://dx.doi.org/10.1002/pros.20303

[11] Rubenstein, M., Hollowell, C.M.P. and Guinan, P. (2011) In LNCaP Cells Enhanced Expression of the Androgen Receptor Compensates for BCL-2 Suppression by Antisense Oligonucleotides. Therapeutic Advances in Urology, 3, 5157.

[12] Rubenstein, M., Anderson, K.M., Tsui, P. and Guinan, P. (2006) Synthesis of Branched Antisense Oligonucleotides Having Multiple Specificities. Treatment of Hormone Insensitive Prostate Cancer. Medical Hypotheses, 67, $1375-1380$. http://dx.doi.org/10.1016/j.mehy.2006.05.055

[13] Rubenstein, M., Dunea, G. and Guinan, P. (1994) Growth Factor Deprivation Therapy Utilizing Antisense Oligonucleotides. Drug News and Perspectives, 7, 517-524.

[14] Rubenstein, M., Mirochnik, Y., Chow, P. and Guinan, P. (1996) Antisense Oligonucleotide Intralesional Therapy of Human PC-3 Prostate Tumors Carried in Athymic Nude Mice. Journal of Surgical Oncology, 62, 194-200. http://dx.doi.org/10.1002/(SICI)1096-9098(199607)62:3<194::AID-JSO9>3.0.CO;2-2 
Scientific Research Publishing (SCIRP) is one of the largest Open Access journal publishers. It is currently publishing more than 200 open access, online, peer-reviewed journals covering a wide range of academic disciplines. SCIRP serves the worldwide academic communities and contributes to the progress and application of science with its publication.

Other selected journals from SCIRP are listed as below. Submit your manuscript to us via either submit@scirp.org or Online Submission Portal.
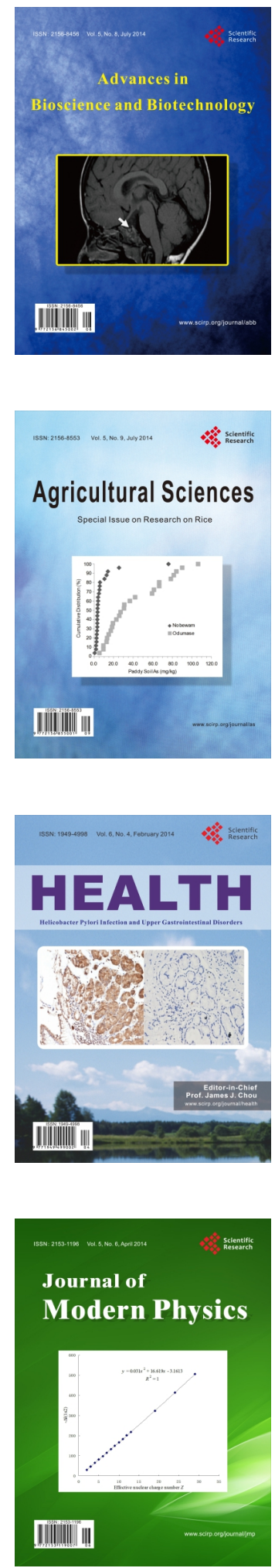
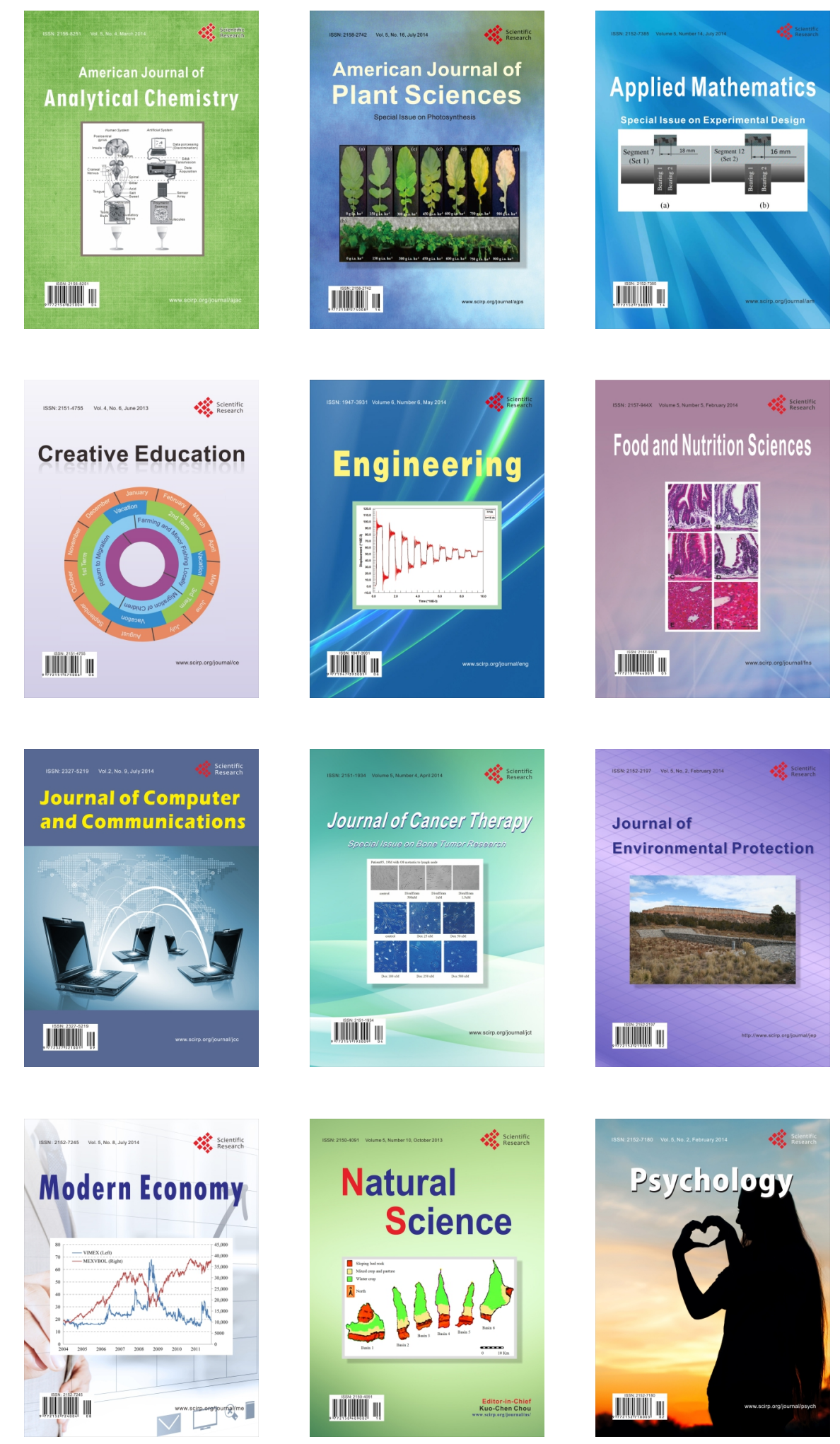